\title{
Percepção dos profissionais de saúde mental sobre maconha
}

\author{
The perception of mental health professionals about marijuana \\ Patrícia Cruz Furtado de Melo', Luciana Roberta Donola Cardoso ${ }^{1}$, André Malbergier²
}

\section{Palavras-chave}

Maconha, percepção, profissionais, saúde mental.

\section{RESUMO}

Objetivo: A percepção sobre a maconha entre profissionais de saúde mental é desconhecida no Brasil. Este estudo teve como objetivo comparar padrões de percepções e de conhecimento sobre maconha entre profissionais da área da saúde mental em três instituições de diferentes perfis. Métodos: Setenta profissionais (psiquiatras, psicólogos, assistentes sociais, terapeutas ocupacionais e enfermeiros) de três serviços da área de saúde mental - Hospital Nossa Senhora de Fátima (hospital filantrópico), CAPS-AD (Serviço público especializado no atendimento de álcool e drogas) e Instituto de Psiquiatria do Hospital das Clinicas da Faculdade de Medicina da Universidade de São Paulo (Serviço acadêmico e assistencial) - foram avaliados por meio de um questionário elaborado pelos pesquisadores, composto de 31 afirmativas. Resultados: Psiquiatras tendem a discordar mais que os outros profissionais quanto à necessidade de tratamento para o uso de maconha e que a maconha traz prejuízos cognitivos. Os profissionais do Hospital Nossa Senhora de Fátima apresentaram percepção mais favorável à proibição de maconha e valorizaram mais os possíveis prejuízos associados ao uso quando comparados aos profissionais das outras duas instituições. Conclusões: A percepção sobre a maconha entre servidores da saúde mental varia de acordo com a profissão e o local de trabalho.

\section{Keywords}

Marijuana, perception, professionals, mental health.

\begin{abstract}
Objective: The perception of marijuana among mental health professionals is unknown in Brazil. The aim of this study was to compare mental health professionals' perception and knowledge standards about marijuana in three institutions with different profiles. Methods: Seventy professionals (psychiatrists, psychologists, social workers, occupational therapists and nurses) from three mental health services - Nossa Senhora de Fátima Hospital (philanthropic hospital), CAPS-AD (public specialized service on treating drugs and alcohol dependents), and the Institute of Psychiatry at the Clinics Hospital, Medical School, University of São Paulo (academic and assistance service) were evaluated through a 31 affirmatives questionnaire created by researchers. Results: Psychiatrists disagree more often than other health professionals on the need to treat marijuana users and on the idea that it causes cognitive damages. The professionals from Nossa Senhora de Fátima Hospital are more in favor of the prohibition of marijuana use and consider more the possible damages associated with its use, when compared to professionals from the other two institutions. Conclusions: The perception of marijuana among mental health providers may vary according to their profession and work place.
\end{abstract}

1 Universidade de São Paulo (USP), Faculdade de Medicina, Departamento de Fisiopatologia Experimental, São Paulo, SP, Brasil. 2 Universidade de São Paulo (USP), Faculdade de Medicina, Departamento de Psiquiatria; Grupo Interdisciplinar de Estudos de Álcool e Drogas (GREA), São Paulo, SP, Brasil.

Endereço para correspondência: André Malbergier

Rua dr Ovídio Pires de Campos, 785

01246-903 - São Paulo, SP, Brasil

E-mail:amalbergier@uol.com.br 


\section{INTRODUÇÃO}

Atualmente, a maconha é a droga ilícita mais usada no Brasil e no mundo. Segundo relatório do órgão das Nações Unidas responsável pelo tema drogas, disponível em 2015, em torno de 181 milhões de pessoas (3,8\% da população mundial entre 15 e 64 anos) usaram maconha no ano anterior à pesquisa!.

No Brasil, de acordo com o II Levantamento Nacional de Álcool e Drogas, 7\% da população adulta já haviam experimentado maconha na vida. No ano anterior à pesquisa, 3\% da população haviam utilizado a droga. Quanto ao uso na adolescência, o estudo mostrou que 4\% da população dessa faixa etária já haviam usado maconha pelo menos uma vez na vida. O uso no último ano foi relatado por $3 \%$ deles. Ainda segundo esse estudo, mais da metade dos usuários, tanto adultos quanto adolescentes, relataram consumir maconha diariamente ${ }^{2}$.

Em outra pesquisa com estudantes universitários no Brasil, os relatos de uso de maconha na vida, no último ano e no último mês foram de 26,1\%, 13,8\% e 9,1\%, respectivamente ${ }^{3}$.

A maconha vem sendo alvo de debates calorosos sobre questões legais, políticas, sociais, médicas e terapêuticas no Brasil e em vários países do mundo ${ }^{4-6}$. Alguns países já descriminalizaram seu consumo ${ }^{7}$ ou a utilizam para fins terapêuticos. O Uruguai foi o primeiro a legalizar e legislar sobre o plantio, comércio e consumo da droga ${ }^{8}$.

O debate sobre o tema também vem gerando interesse crescente em estudar os potenciais prejuízos à saúde associados ao consumo de maconha. Os estudos na área destacam os prejuízos nas áreas psiquiátrica (transtornos ansiosos, depressivos e psicóticos) e cognitiva ${ }^{10}$.

Apesar das evidências desses prejuízos, as percepções sobre o uso de maconha e suas consequências variam muito entre profissionais da área da saúde. Essa percepção tem grande importância, já que eles são responsáveis por participar da elaboração de políticas públicas, pelas intervenções terapêuticas e possíveis aconselhamentos ${ }^{11}$.

O número de estudos sobre as percepções e opiniões dos profissionais de saúde sobre o tema não parece refletir a sua importância. Muito poucas pesquisas sobre o tema foram publicadas.

Em um estudo pioneiro, na década de 1970, 45 médicos foram entrevistados e a opinião que prevaleceu foi a de que a maconha e o álcool são as drogas que acarretam menor prejuízo. Por outro lado, quando questionados sobre consequências psiquiátricas, a maconha foi considerada mais perigosa que o tabaco. Apesar disso, 71\% não acreditavam que a maconha causasse psicose. Na opinião dos médicos entrevistados, a maconha seria uma droga que apresenta prejuízos nos aspectos social e psicológico, porém não seria alvo de grandes preocupações quando comparada às outras drogas $^{12}$.
Outra pesquisa, realizada mais recentemente, com 303 médicos psiquiatras da universidade da Califórnia e clínicas afiliadas, mostrou que os médicos mais jovens, que já fizeram uso de álcool, tabaco e tranquilizantes, menos religiosos e politicamente mais liberais não viam problemas no uso da maconha e apoiavam a legalização. Por outro lado, os médicos mais conservadores apoiavam a sua criminalização ${ }^{13}$.

O fato dos médicos com diferentes características sociodemográficas terem diferentes opiniões pode indicar que elas podem estar mais baseadas em aspectos pessoais do que em conhecimentos técnicos.

No cenário atual de debates sobre a maconha e levando em consideração a existência de pouquíssimos estudos internacionais e a ausência de pesquisas no Brasil sobre esse tema, esta pesquisa visa avaliar a percepção sobre a maconha entre profissionais da área de saúde mental em três instituições de diferentes perfis na cidade de São Paulo, Brasil.

Nossa hipótese é de que fatores sociodemográficos e institucionais influenciam a percepção dos profissionais de saúde mental sobre maconha.

\section{MÉTODOS}

\section{Local e período}

Esta pesquisa foi realizada em três instituições de perfis distintos: hospital psiquiátrico Nossa Senhora de Fátima (NSF), Centro de Atenção Psicossocial para atendimento de problemas relacionados ao uso de álcool e drogas (CAPS-AD) e Instituto de Psiquiatria do Hospital das Clínicas da Faculdade de Medicina da Universidade de São Paulo (IPq), no período de setembro a dezembro de 2012. A escolha das instituições foi com a intenção de avaliar se o perfil institucional é uma variável capaz de influenciar os resultados.

O NSF é uma instituição filantrópica coordenada por freiras ligadas à igreja católica e conta com uma equipe multidisciplinar (psiquiatras, psicólogos, assistentes sociais, terapeutas ocupacionais e enfermeiros). O hospital oferece atendimento a mulheres com transtornos psiquiátricos graves, com atendimento ambulatorial e internação.

O CAPS-AD é um serviço ambulatorial público especializado no atendimento de pessoas com problemas decorrentes do abuso de álcool e outras drogas em diferentes níveis de cuidado: intensivo (diariamente), semi-intensivo (de duas a três vezes por semana) e não intensivo (uma vez por semana).

O IPq é uma instituição acadêmica dentro do Complexo Hospital das Clínicas associado a Faculdade de Medicina da Universidade de São Paulo, Brasil, com ênfase em ensino, pesquisa e assistência (atendimento ambulatorial, internações e hospital dia). 


\section{Sujeitos}

Os profissionais da área da saúde mental que trabalhavam nas três instituições descritas acima foram convidados a responder, sem precisar se identificar, a um questionário de autopreenchimento que continha 31 afirmações sobre o tema. Esse questionário foi desenvolvido pelos pesquisadores, já que a investigação da literatura, até o momento, não encontrou questionários padronizados para esse fim (vide limitações). Os profissionais deveriam avaliar as 31 afirmações por meio de um escore que variava em uma escala de 1 a 7 , no qual 1 significava discordo plenamente e 7 concordo plenamente (Tabela 1).

Participaram da pesquisa 70 profissionais de cinco áreas da saúde mental: psiquiatras, psicólogos, assistentes sociais, terapeutas ocupacionais e enfermeiros.

Foram entrevistados todos os profissionais listados acima que trabalhavam na época no NSF e do CAPS-AD. No IPq, devido ao grande número de profissionais das cinco áreas estudadas que atuam no serviço, os participantes foram selecionados aleatoriamente por meio de sorteio a partir de uma lista de todos os profissionais de cada área que trabalham nessa instituição.

Não houve recusa de participação de nenhum profissional do CAPS e NSF. Quatro médicos do HC, inicialmente sorteados, receberam o questionário e não o devolveram. Nessa situação, após o término do prazo de uma semana, foram chamados os médicos que vinham a seguir em uma lista de 24 sujeitos sorteados no início do estudo.

\section{Aspectos éticos}

O projeto de pesquisa foi aprovado pelos Comitês de Ética em Pesquisa do Hospital das Clínicas da Faculdade de Medicina da Universidade de São Paulo e das outras duas

Tabela 1. Questionário sobre a percepção sobre a maconha entre profissionais de saúde mental

\begin{tabular}{|c|c|}
\hline 1. A maconha é a droga ilícita mais usada em nosso meio & 1234567 \\
\hline 2. A maconha possui ação direta no sistema nervoso central & 1234567 \\
\hline 3. A maconha pode ser considerada uma droga leve & 1234567 \\
\hline 4. A maconha produz dependência & 1234567 \\
\hline 5. Indivíduos dependentes da maconha podem ter abstinência da droga quando diminuem ou param de usar & 1234567 \\
\hline 6. A maconha pode ser uma porta de entrada para outras drogas & 1234567 \\
\hline 7. Utilizar maconha pode controlar a fissura por outras drogas & 1234567 \\
\hline 8. 0 uso da maconha pode desencadear depressão e outros transtornos de humor & 1234567 \\
\hline 9. 0 uso da maconha pode desencadear transtorno de ansiedade & 1234567 \\
\hline 10. 0 uso da maconha pode desencadear transtorno psicótico & 1234567 \\
\hline 11. 0 uso da maconha e derivados para fins terapêuticos deveria ser proibido & 1234567 \\
\hline 12. Existe tratamento para dependência de maconha & 1234567 \\
\hline 13. Um usuário de maconha deve ser encaminhado para tratamento & 1234567 \\
\hline 14. 0 uso da maconha deve ser tolerado & 1234567 \\
\hline 15. 0 uso da maconha deve ser descriminalizado & 1234567 \\
\hline 16. A maconha produz prejuízos cognitivos (memória, atenção, funções executivas) & 1234567 \\
\hline 17. 0 álcool é mais prejudicial que a maconha & 1234567 \\
\hline 18. Fumar maconha faz parte da adolescência & 1234567 \\
\hline 19. Maconha é menos prejudicial que o tabaco & 1234567 \\
\hline 20. Maconha pode ser usada para tratamento de dependência de outras drogas & 1234567 \\
\hline 21. 0 tratamento para maconha é parecido com o tratamento para outras drogas & 1234567 \\
\hline 22. A maconha traz prejuízos físicos, psicológicos e sociais & 1234567 \\
\hline 23. Os pais deveriam tolerar 0 uso de maconha em casa & 1234567 \\
\hline 24. 0 risco de ser pego pela polícia fumando maconha é maior do que da própria droga & 1234567 \\
\hline 25. Não trataria de um indivíduo que só usa maconha & 1234567 \\
\hline 26. Não internaria dependentes de maconha se só usassem maconha & 1234567 \\
\hline 27. 0 uso da maconha deve ser legalizado & 1234567 \\
\hline 28. Existem riscos do uso da maconha na gravidez & 1234567 \\
\hline 29. Fumante passivo (exposto a fumaça) de maconha não apresenta riscos & 1234567 \\
\hline 30. Considera-se careta e conservador quem "fala mal" da maconha & 1234567 \\
\hline 31. Considero-me bem informado sobre a maconha & 1234567 \\
\hline
\end{tabular}


instituições envolvidas. Todos os sujeitos assinaram um termo de consentimento que explicava a pesquisa e garantia o sigilo das informações obtidas por meio do questionário.

\section{Análise estatística}

Inicialmente, a amostra foi descrita de acordo com o gênero, idade, número de filhos, religião, profissão, local de trabalho, uso de maconha e outras substâncias. As variáveis foram descritas por frequência (n), porcentagem (\%), médias e desvio-padrão (SD). Foram avaliadas as associações entre percepção sobre maconha pelos profissionais e as variáveis acima listadas.

Para cada afirmação, conforme consta na metodologia, as opiniões eram avaliadas por meio de um escore que podia variar de 1 a 7 . As análises consistiram da comparação entre as médias dos escores das opiniões para cada variável estudada.

Exemplo: Comparação entre casados e não casados em relação à opinião sobre a afirmativa 2: A maconha possui ação direta no sistema nervoso central. Os casados tiveram média de 6,8 e os não casados, de 6,6. Nesse caso, a diferença não foi significativa.

Para as análises, foram utilizados os testes de qui-quadrado $\left(X^{2}\right)$ de Pearson e o exato de Fisher. O nível de significância considerado neste artigo foi de 0,05. Todas as análises foram realizadas pelo software SPSS (Statistical Package for Social Science) para Windows, versão 16.0.

\section{RESULTADOS}

\section{Característica da amostra - Aspectos sociodemográficos}

A amostra foi constituída por 70 profissionais de saúde mental, 23 (32,9\%) do NSF, 23 (32,9\%) do CAPS-AD e 24 (34,3\%) do IPq. Dez (14,3\%) eram assistentes sociais, 10 (14,3\%), enfermeiros, 29 (41,4\%), médicos, 11 (15,7\%), psicólogos e 10 $(14,3 \%)$, terapeutas ocupacionais. Desses, somente um não havia atendido usuários de drogas. Quarenta e nove profissionais eram do sexo feminino (70\%) e 21, do sexo masculino (30\%). A idade média foi de 34 anos. Dos profissionais entrevistados, 41 (58,6\%) não tinham filhos. Houve predominância dos solteiros ( $37-52,8 \%$ ), seguidos pelos casados (27 - 38,6\%), separados $(4-5,7 \%)$ e viúvos $(2-2,9 \%)$.

Trinta e seis $(51,4 \%)$ profissionais eram católicos, 8 (11,4\%), evangélicos, 7 (10\%), espíritas, 3 (4,3\%), judeus e 2 $(2,9 \%)$, budistas. Quatorze (20\%) profissionais relataram não ter nenhuma religião.

As frequências das variáveis distribuição dos profissionais, gênero, estado civil, religião, ter filhos e se atendem ou não usuários de drogas foi semelhante nas três instituições pesquisadas $(p>0,05)$.

Dos profissionais, 16 (22,9\%) já haviam usado maconha na vida. O relato de consumo de maconha foi mais frequen- te entre os profissionais do HC $(62,5 \%)$ do que entre os do $\operatorname{HNSF}(12,5 \%)(p=0,02)$. Vale ressaltar que 32 sujeitos $(45,7 \%)$ não responderam a essa questão.

Os homens consideravam-se mais bem informados sobre a maconha do que as mulheres (Afirmação 31) ( $p=0,01$ ). Profissionais que têm filhos concordam mais com a afirmativa Um usuário de maconha deve ser encaminhado para tratamento, quando comparados aos que não têm filhos.

O fato de já ter feito uso de droga também influenciou a opinião dos profissionais sobre as seguintes afirmativas: A maconha possui ação direta no SNC. Nessa afirmativa, quem já fez uso de droga concorda mais do que quem não fez ( $p<0,001)$. O uso de maconha deve ser tolerado: nessa afirmativa quem nunca fez uso de droga discorda mais quando comparado a quem já fez $(p=0,05)$. Fumar maconha faz parte da adolescência: quem já fez uso de droga concorda mais com essa afirmativa quando comparado a quem não fez ( $p$ $=0,04$ ). Não internaria dependentes de maconha se só usassem maconha: quem nunca fez uso de droga discorda mais dessa questão do que quem já fez uso $(p=0,05)$.

\section{Instituição e percepção sobre maconha}

A tabela 2 mostra a análise univariada da relação entre instituição e percepção sobre maconha. Foram apresentadas nessa tabela apenas as associações entre as afirmações e a instituição em que o profissional trabalha com nível de significância até 0,1 . Os profissionais do NSF discordaram mais que os de outras instituições ao avaliar as seguintes afirmativas: Fumar maconha faz parte da adolescência, Maconha pode ser usada para tratamento de dependências de outras drogas e Não trataria de um indivíduo que só usa maconha. A avaliação da afirmação: Considera-se careta e conservador quem "fala mal" da maconha apresentou diferença marginal $(p=0,06)$. Os profissionais do NSF concordaram mais (significância entre 0,05 e 0,1) do que os das outras instituições na avaliação das afirmações: Existe tratamento para dependência de maconha e A maconha produz prejuizos cognitivos.

\section{Profissionais e percepção sobre maconha}

Na tabela 3, é possível observar a relação entre a categoria profissional e a percepção sobre maconha. Os profissionais foram agrupados em médicos e não médicos.

Não médicos concordaram mais com a afirmação: Um usuário de maconha deve ser encaminhado para tratamento ( $p=0,01)$, que os médicos. Já para outras duas afirmações, as diferenças foram marginais $(p=0,08)$. Não médicos concordam mais que A maconha produz dependência e A maconha produz prejuízos cognitivos.

\section{Religião e percepção sobre maconha}

A tabela 4 mostra a relação entre religião e percepção sobre maconha. Quem tem religião discorda mais da afirmação: Fumar maconha faz parte da adolescência. Por outro lado, 
quem tem religião concorda mais (nível de significância marginal $-p=0,06)$ com a afirmação: $A$ maconha traz prejuízos físicos, psicológicos e sociais.

\section{DISCUSSÃO}

O objetivo desta pesquisa foi avaliar as opiniões e percepções de profissionais da área da saúde mental sobre a maconha em três instituições de perfis diferentes (Hospital Psiquiátrico Nossa Senhora de Fátima, CAPS-AD e Hospital das Clínicas).

Os resultados desta pesquisa mostram que algumas características sociodemográficas foram associadas a opiniões diferentes em relação a algumas afirmativas. Profissionais com filhos concordaram mais com a afirmação de que o uso de maconha pode ser objeto de busca de tratamento. Sujeitos que referiram já ter usado drogas parecem ter opiniões mais favoráveis e "liberais" quanto ao uso de maconha.

Quanto à religião, quem tem alguma religião discorda mais de que maconha faz parte da adolescência.

A influência das características sociodemográficas na opinião sobre a maconha em médicos já havia sido aponta- da em um estudo antigo. Os pesquisadores observaram que os médicos mais velhos, casados e que praticam alguma religião são mais conservadores em relação à maconha ${ }^{13}$.

No nosso estudo, a variável idade não pareceu influenciar as opiniões sobre a maconha, diferente da pesquisa acima citada. A média de idade de 34 anos (relativamente jovem) e a pouca variação da faixa etária em nossa amostra podem estar associadas a não identificação de alguma influência da faixa etária nas respostas dos participantes.

A religião é uma variável que tem sido constantemente apontada como fator protetor em relação a uso de drogas na população geral ${ }^{14}$ e também como influência na percepção e opinião sobre a questão entre profissionais de saúde ${ }^{13}$. A religião dos médicos também já foi considerada um fator de influência nas opiniões e atitudes em relação a outros temas na área da saúde como aborto ${ }^{15}$ e situações de final de vida ${ }^{16}$ e a atitudes proibitivas em relação ao uso de drogas ${ }^{17}$.

Quando os profissionais das três diferentes instituições foram comparados, observamos que os que trabalhavam no NSF mostraram posicionamento contra a descriminalização da maconha e viam a maconha como fonte de maiores prejuízos quando comparados aos de outras instituições.

Tabela 2. Diferenças na percepção sobre a maconha entre profissionais das três instituições estudadas

\begin{tabular}{|c|c|c|c|c|c|c|c|c|c|}
\hline \multirow{3}{*}{ Questão } & \multicolumn{6}{|c|}{ Instituiçäo } & \multirow{2}{*}{\multicolumn{2}{|c|}{ Total }} & \multirow[b]{3}{*}{ P-Valor } \\
\hline & \multicolumn{2}{|c|}{ CAPS } & \multicolumn{2}{|c|}{$\mathbb{P P q}$} & \multicolumn{2}{|c|}{ NSF } & & & \\
\hline & $\mathrm{N}$ & Média & $\mathrm{N}$ & Média & $\mathrm{N}$ & Média & $\mathrm{N}$ & Média & \\
\hline Fumar maconha faz parte da adolescência & 23 & $1,8(1,28)$ & 24 & $2,5(1,91)$ & 23 & $1,3(1,19)$ & 70 & $1,9(1,55)$ & 0,01 \\
\hline $\begin{array}{l}\text { Maconha pode ser usada para tratamento de dependência de } \\
\text { outras drogas }\end{array}$ & 23 & $2,9(2,17)$ & 24 & $2,4(1,98)$ & 23 & $1,3(0,88)$ & 70 & $2,2(1,85)$ & 0,01 \\
\hline Não trataria de um indivíduo que só usa maconha & 23 & $1,8(1,48)$ & 24 & $1,5(1,21)$ & 23 & $1,1(0,62)$ & 70 & $1,5(1,17)$ & 0,05 \\
\hline
\end{tabular}

CAPS: Centro de Atenção Psicossocial para atendimento de problemas relacionados ao uso de álcool e drogas; IPq: Instituto de Psiquiatria do Hospital das Clínicas da Faculdade de Medicina da Universidade de São Paulo; NSF: Hospital Psiquíátrico Nossa Senhora de Fátima.

Tabela 3. Diferenças de percepções sobre a maconha entre médicos e não médicos

\begin{tabular}{|c|c|c|c|c|c|c|c|}
\hline & \multicolumn{4}{|c|}{ Profissão } & \multirow{2}{*}{\multicolumn{2}{|c|}{ Total }} & \multirow[b]{3}{*}{ P-valor } \\
\hline & \multicolumn{2}{|c|}{ Não médicos } & \multicolumn{2}{|c|}{ Médicos } & & & \\
\hline & N & Média & $\mathrm{N}$ & Média & $\mathrm{N}$ & Média & \\
\hline A maconha produz dependência & 39 & $6,6(0,71)$ & 29 & $6,0(1,30)$ & 68 & $6,3(1.03)$ & 0,08 \\
\hline Um usuário de maconha deve ser encaminhado para tratamento & 41 & $6,2(1,26)$ & 29 & $5,3(1,78)$ & 70 & $5,8(1,84)$ & 0,01 \\
\hline $\begin{array}{l}\text { A maconha produz prejuízos cognitivos (memória, atenção, funções } \\
\text { executivas) }\end{array}$ & 41 & $6,8(0,44)$ & 29 & $6,5(0,78)$ & 70 & $6,7(0,61)$ & 0,08 \\
\hline
\end{tabular}

Tabela 4. Diferença de percepção sobre a maconha entre profissionais com e sem religião

\begin{tabular}{|c|c|c|c|}
\hline \multirow{3}{*}{ Questão } & \multicolumn{2}{|c|}{ Religiäo } & \multirow{3}{*}{ P-valor } \\
\hline & Não $(N=12)$ & $\operatorname{Sim}(N=58)$ & \\
\hline & Média & Média & \\
\hline Fumar maconha faz parte da adolescência & $2,8(1,71)$ & $1,8(1,48)$ & 0,03 \\
\hline $\begin{array}{l}0 \text { tratamento para maconha é parecido com o tratamento para } \\
\text { outras drogas }\end{array}$ & $5,8(1,78)$ & $6,6(0,98)$ & 0,06 \\
\hline
\end{tabular}


As razões para essa associação podem ser: o NSF, por ser uma instituição religiosa, pode ter uma política mais "conservadora" quanto aos usuários de maconha e isso é refletido nas opiniões de seus profissionais ou os profissionais mais religiosos buscam mais esse hospital para trabalhar devido a crenças comuns. Quanto à segunda hipótese, os resultados não mostraram diferenças na frequência de profissionais que referem ter alguma religião entre as três instituições. Todavia, esta pesquisa não avaliou a prática religiosa.

Quando os profissionais foram agrupados em categorias, observou-se que os não médicos concordaram mais que os médicos na avaliação das afirmativas que ressaltam a necessidade de tratamento para usuários de maconha e que essa droga produz dependência e gera prejuízos cognitivos.

Como esta pesquisa avaliou somente médicos psiquiatras e essa especialidade parece ter um perfil mais tolerante em relação a drogas quando comparada às outras, esta comparação entre médicos e não médicos, realizada neste estudo, pode não ser generalizável para a população médica como um todo. Como exemplo, um estudo que comparou a atitude e opiniões de 208 médicos de diferentes especialidades sobre álcool e drogas ${ }^{18}$ observou que os médicos psiquiatras tiveram respostas com conteúdos mais "liberais" em relação às drogas e seu tratamento. Os autores sugerem que, por ser uma especialidade que trabalha diretamente com a parte farmacológica e comportamental do paciente, sua visão seja diferenciada em relação às outras especialidades. O fato de ter mais conhecimento técnico do problema faria com que tal profissional tivesse uma percepção menos estigmatizada do problema com drogas.

Outra hipótese para explicar as diferenças entre médicos e não médicos encontradas no nosso estudo seria uma maior tolerância ao consumo da droga por parte dos médicos devido ao fato de a maconha estar sendo vista como forma de tratamento para diversas patologias, gerando o conceito de uso terapêutico ${ }^{19,20}$, atualmente permitido pelo Conselho Federal de Medicina. Também recentemente, no Brasil, a Anvisa (Agência Nacional de Vigilância Sanitária) liberou empresas a registrarem produtos com canabidiol e tetrahidrocanabinol como princípios ativos e a prescrição, por parte dos médicos, das medicações que serão registradas. Essa liberação deverá promover a necessidade de prover informações sobre o tema para a classe médica e outros profissionais de saúde, gerando maior conhecimento sobre o tema. Nessa linha, um estudo irlandês com médicos daquele país demonstrou que o nível de formação e treinamento na área de substâncias de abuso/dependência esteve fortemente associado a uma postura mais tolerante e favorável ao uso médico de cannabis $^{21}$. Diante disso, alguns estudiosos da área sugerem a criação de uma disciplina nas faculdades de Medicina, denominada "Cannabis Curriculum"22.

Nosso estudo avaliou médicos e outros quatro tipos de profissionais da área de saúde mental: enfermeiros, assis- tentes sociais, psicólogos e terapeutas ocupacionais. A discussão tem se focado mais em médicos, já que não foram encontrados, exceto alguns com enfermeiros, estudos que analisaram o tema em não médicos.

Em um desses estudos que incluiu médicos e enfermeiros, os resultados mostraram que menos de $1 / 3$ dos médicos generalistas considerava suas habilidades para lidar com dependentes de maconha aceitáveis. Apesar de parecer baixa, essa porcentagem foi ainda menor entre enfermeiros $^{23}$. Uma ampla revisão publicada em 2013 sobre o tema também apresenta mais dados sobre enfermeiros e poucos sobre psicólogos, terapeutas ocupacionais e assistentes sociais $^{24}$

Uma implicação prática deste estudo seria: será que as percepções e opiniões podem prejudicar ou beneficiar o paciente que está recebendo tratamento dos profissionais?

Alguns estudos parecem mostrar que as atitudes e opiniões podem sim influenciar no tratamento dos dependentes. Os profissionais de saúde são formadores de opiniões e, segundo Clay et al., a percepção sobre o uso de drogas e suas consequências entre profissionais da área da saúde têm importância significativa, já que eles são responsáveis por intervenções e possível aconselhamento que podem mudar o curso da utilização de drogas ${ }^{25}$. Outros estudos também apontam que o posicionamento e o conhecimento do profissional de saúde sobre o tema são fundamentais para a eficácia do tratamento do paciente dependente de drogas ${ }^{18,26,27}$. As atitudes negativas dos profissionais de saúde podem gerar problemas na comunicação entre o profissional e o paciente, baixo interesse do profissional para investigação do diagnóstico da dependencia e de complicações físicas dela e prejudicar a aliança terapêutica ${ }^{28}$.

No caso específico da maconha, os dependentes dessa droga têm algumas particularidades quando comparado aos outros dependentes. Eles mostram maior nível de ambivalência quanto aos prejuízos da droga e menor motivação para mudanças de comportamentos, além de referirem ter menos necessidade de tratamento. Quando eles têm contato com os profissionais de saúde, a abordagem destes mostra-se ainda mais importante para atraí-los para tratamento e estimulá-los a ter cuidados com sua saúde ${ }^{29,30}$.

Esse cenário de grande variação de opiniões e atitudes quanto ao consumo de maconha estimula reflexão e o desenvolvimento de estratégias para abordar a questão. Educação e treinamento de profissionais de saúde parecem estar associados à diminuição de estigma, maior uniformidade de conhecimento e atitudes em equipes de saúde mental que trabalham com dependentes ${ }^{31}$. As limitações de conhecimento sobre o tema parecem ir além dos profissionais de saúde mental propriamente dito e se estendem às ações primárias de saúde. Estudo com agentes comunitários de saúde também ressalta a necessidade de cursos de capacitação e educação permanente na área ${ }^{32}$. 
Algumas limitações deste estudo podem ser apontadas. A amostra é relativamente pequena e o número de sujeitos não foi obtido por meio de um cálculo amostral rigoroso. Essas limitações decorreram do número de profissionais relativamente pequeno no NSF e no CAPS-AD e da falta de estudos que nos ajudassem a estimar, de forma mais acurada, o tamanho da amostra. A ausência de questionários padronizados para a investigação do tema gerou a necessidade da criação, por parte dos autores, do instrumento utilizado nesta pesquisa. Tal procedimento foi utilizado pela grande maioria dos estudos discutidos neste artigo ${ }^{21}$. A escolha das instituições por conveniência para representar os diferentes modelos assistenciais pode também não representar todos os profissionais de saúde mental e, assim, prejudicar a generalização dos resultados obtidos. Cabe dizer, finalizando, que, apesar dessas limitações, o presente estudo tem como mérito abordar, de maneira objetiva, um tema ainda muito pouco presente na discussão sobre a maconha em nosso país. Poucos estudos e debates são focados na qualidade e quantidade de informações que os profissionais de saúde mental têm sobre essa questão.

\section{CONCLUSÕES}

Este estudo evidencia que a opinião dos profissionais de saúde sobre a maconha e consequentemente sobre o usuário pode ser influenciada por variáveis pessoais como as sociodemográficas. Tal influência pode aumentar a chance de abordagens inapropriadas, gerando risco de prejuízos aos pacientes.

\section{CONTRIBUIÇÕES INDIVIDUAIS}

Patrícia Melo - Responsável pela concepção e planejamento da pesquisa, aplicação dos questionários e redação do manuscrito.

Luciana Cardoso - Responsável pela análise estatística e elaboração do manuscrito.

Andre Malbergier - Responsável pela concepção e supervisão da pesquisa, análise dos dados e redação final do manuscrito.

\section{CONFLITO DE INTERESSES}

Os autores declaram não ter conflitos de interesses.

\section{REFERÊNCIAS}

1. United Nations Office on Drug and Crime - UNODC. World Drug Report 2015. Disponível em: www.unodc.org. Acesso em: 10 ago 2018.
2. Instituto Nacional de Ciência e Tecnologia para Políticas Públicas do Álcool e Outras Drogas - INPAD. II Levantamento Nacional de Álcool e Drogas. Disponível em: http://inpad.org.br/ lenad/resultados/maconha/. Acesso em: 10 ago 2018.

3. Andrade AG, Duarte PCAV, Oliveira, LG (Orgs.). I Levantamento Nacional sobre 0 uso de Álcool, Tabaco e Outras Drogas entre Universitários das 27 Capitais Brasileiras. Brasília: Secretaria Nacional de Política Sobre Drogas; 2010.

4. Kiepper A, Esher A. Regulation of marijuana by the Brazilian Senate: a public health issue. Cad Saúde Pública. 2014;30(8):1588-90.

5. Room R. Legalizing a market for cannabis for pleasure: Colorado, Washington, Uruguay and beyond. Addiction. 2014;109(3):345-51.

6. Hall W, Weier M. Assessing the public health impacts of legalizing recreational cannabis use in the USA. Clin Pharmacol Ther. 2015;97(6):607-15.

7. Ogrodnik M, Kopp P, Bongaerts X, Tecco JM. An economic analysis of different cannabis decriminalization scenarios. Psychiatr Danub. 2015;27(Suppl 1):S309-14.

8. Wade L. South America. Legal highs make Uruguay a beacon for marijuana research. Science. 2014;344(6189):1217.

9. Radhakrishnan R, Wilkinson ST, D'Souza DC. Gone to Pot - A Review of the Association between Cannabis and Psychosis. Front Psychiatry. 2014;22(5):54.

10. Thames AD, Arbid N, Sayegh P. Cannabis use and neurocognitive functioning in a nonclinical sample of users. Addict Behav. 2014;39(5):994-9.

11. Breton AR, Taira DA, Burns E, O'Leary J, Chung RS. Follow-up services after an emergency department visit for substance abuse. Am J Manag Care. 2007;13(9):497-505.

12. Lieff J, Avorn J, Cadell H, Anderson H, Gorlin P, Kennedy S, et al. Attitudes of the medical profession toward drug abuse. Am J Psychiatry. 1973;63:1035-39.

13. Linn LS, Yager J, Leak B. Physicians' attitudes toward the legalization of marijuana use. West J Med. 1989;150 (6):714-7.

14. Gmel G, Mohler-Kuo M, Dermota P, Gaume J, Bertholet N, Daeppen JB, et al. Religion is good, belief is better: religion, religiosity, and substance use among young Swiss men. J Subst Use Misuse. 2013;48(12):1085-98.

15. Marván ML, Álvarez del Río A, Campos Z. On abortion: exploring psychological meaning and attitudes in a sample of Mexican gynecologists. Dev World Bioeth. 2014;14(1):29-36.

16. Bateman LB, Clair JM. Physician religion and end-of-life pediatric care: a qualitative examination of physicians perspectives. Narrat Inq Bioeth. 2015;5(3):251-69.

17. Richard AJ, Bell DC, Carlson JW. Individual religiosity, moral community, and drug user treatment. J Sci Study Relig. 2000;39:240-6.

18. Lev-Ran S, Adler L, Nitzan U, Fennig S. Attitudes towards nicotine, alcohol and drug dependence among physicians in Israel. J Subst Abuse Treat. 2013;44(1):84-9.

19. Wilkins MR; Working Party on Cannabis and Cannabis-based Medicines. Cannabis and cannabis-based medicines: potential benefits and risks to health. Clin Med (Lond). 2006;6(1):16-8.

20. Juurlink DN. Medicinal cannabis: time to lighten up? CMAJ. 2014;186(12):897-8.

21. Crowley D, Collins C, Delargy I, Laird E, Van Hout MC. Irish general practitioner attitudes toward decriminalisation and medical use of cannabis: results from a national survey. Harm Reduct J. 2017;14:4.

22. Ware MA, Ziemianski D. Medical education on cannabis and cannabinoids: Perspectives, challenges, and opportunities. Clin Pharmacol Ther. 2015;97(6):548-50.

23. Norberg MM, Gates P, Dillon P, Kavanagh DJ, Manocha R, Copeland J. Screening and managing cannabis use: comparing GP's and nurses' knowledge, beliefs, and behavior. Subst Abuse Treat Prev Policy. 2012;7:31.

24. Van Boekel LC, Brouwers EP, Van Weeghel J, Garretsen HF. Healthcare professionals' regard towards working with patients with substance use disorders: comparison of primary care, general psychiatry and specialist addiction services. Drug Alcohol Depend. 2014;1(134):92-8.

25. Clay SW, Allen J, Parran T. A review of addiction. Postgrad Med. 2008;31(120):E01-7.

26. Brener L, Von Hippel W, Kippax S, Preacher KJ. The role of physician and nurse attitudes in the health care of injecting drug users. Subst Use Misuse. 2010;45(7-8):1007-18.

27. Van Boekel LC, Brouwers EP, Van Weeghel J, Garretsen HF. Stigma among health professionals towards patients with substance use disorders and its consequences for healthcare delivery: systematic review. Drug Alcohol Depend. 2013;131(1-2):23-35. 
28. Palmer RS, Murphy MK, Piselli A, Ball SA. Substance user treatment dropout from client and clinician perspectives: a pilot study. Subst Use Misuse. 2009;44(7):1021-38

29. Stewart $D$. Drug use and perceived treatment need among newly sentenced prisoners in England and Wales. Addiction. 2009;104(2):243-7.

30. Mariani JJ, Cheng WY, Bisaga A, Sullivan M, Carpenter K, Nunes EV, et al. Comparison of clinical trial recruitment populations: treatment-seeking characteristics of opioid, cocaine, and cannabis-using participants. J Subst Abuse Treat. 2011;40(4):426-30.
31. Howard V, Holmshaw J. Inpatient staff perceptions in providing care to individuals with c0-occurring mental health problems and illicit substance use. J Psychiatr Ment Health Nurs. 2010;17(10):862-72.

32. Araujo LF, Castanha AR, Barros APR, Castanha CR. Estudo das representações sociais da maconha entre agentes comunitários de saúde. Ciênc Saúde Coletiva. 2006;11(3): 827-36. 